\title{
Smooth braneworld in 6-dimensional asymptotically AdS spacetime
}

\author{
Jun-Jie Wan, ${ }^{a, b}$ Zheng-Quan Cui, ${ }^{a, b}$ Wen-Bin Feng ${ }^{a, b}$ and Yu-Xiao Liu ${ }^{a, b, c, 1}$ \\ ${ }^{a}$ Institute of Theoretical Physics \\ and Research Center of Gravitation, Lanzhou University, \\ Lanzhou 730000, P.R. China \\ ${ }^{b}$ Lanzhou Center for Theoretical Physics and \\ Key Laboratory of Theoretical Physics of Gansu Province, Lanzhou University, \\ Lanzhou 730000, P.R. China \\ ${ }^{c}$ Key Laboratory for Magnetism and Magnetic Materials of the MOE, \\ Lanzhou University, Lanzhou 730000, P.R. China \\ E-mail: wanjj@19lzu.edu.cn, cuizhq2017@lzu.edu.cn, \\ fengwb18@lzu.edu.cn, liuyx@lzu.edu.cn
}

ABSTRACT: In this paper, we investigate a six-dimensional smooth thick braneworld model which contains a compact extra dimension and an infinite large one. The braneworld is generated by a real scalar field with a $\phi^{6}$ potential and the bulk is an asymptotically $\mathrm{AdS}_{6}$ spacetime. The geometry achieves the localization of the free $\mathrm{U}(1)$ gauge field, which is a problem in five-dimensional Randall-Sundrum-like models. In addition, we analyze the stability of the braneworld system and the localization of the graviton.

KeYwords: Large Extra Dimensions, Classical Theories of Gravity

ArXiv EPRINT: 2010.05016

\footnotetext{
${ }^{1}$ Corresponding author.
} 


\section{Contents}

1 Introduction 1

2 Setup 3

3 Two braneworld solutions in six-dimensional asymptotically AdS spacetime

3.1 Solution 1

3.2 Solution 2

4 Linear tensor perturbations and localization of the graviton $\quad 7$

5 Localization of scalar fields and vector fields $\quad \mathbf{1 2}$

$\begin{array}{lll}5.1 & \text { Scalar fields } & 12\end{array}$

$\begin{array}{ll}5.2 \text { Vector fields } & 14\end{array}$

6 Conclusions and discussions $\quad 19$

\section{Introduction}

As an extra-dimensional theory, the braneworld scenario provides an alternative framework for outstanding issues, such as the hierarchy problem [1-4] and the cosmological constant problem $[5,6]$. Hence, the braneworld theory has attracted extensive attention and various braneworld models have been proposed.

The braneworld theory must be consistent with experiments. The Newtonian potential indicates there are only three large spatial dimensions. Therefore, it is natural to compact extra dimensions into a small spatial volume, which needs high energy to observe. One of the famous theories is the Kaluza-Klein (KK) theory [7, 8]. However, Rubakov and Shaposhnikov proposed the possibility of noncompact extra dimensions, known as the domain wall model $[9,10]$. The model shows that we live inside a domain wall generated by a scalar field in a five-dimensional flat spacetime, but the Newtonian potential is not recovered. Later, Randall and Sundrum (RS) proposed the RS-2 model where the Newtonian potential can be recovered on the brane even though the extra dimension is infinite [4]. Various extensions of the RS-2 model have been investigated [11-17].

The RS-2 model assumes that particles in the standard model (SM) of particle physics are localized on a hypersurface (3-brane) in the bulk [4]. A more realistic braneworld model should provide a reasonable dynamic interpretation for this assumption. Combining the RS-2 model and the domain wall model, braneworld models with thickness and inner structure were proposed as thick branes $[11,12,18,19]$. In this scenario, various matter fields distribute in the bulk. The SM fields corresponding to zero modes of various bulk 
matter fields and interactions should be reproduced on the brane [20], so it is important to investigate the localization of various bulk matter fields.

For five-dimensional RS-like braneworld models, ref. [21] indicates that not all free boson fields are localized on the brane. It shows that a free massless scalar field and graviton are localized but a free $\mathrm{U}(1)$ gauge field is not. Hence, the localization mechanisms of gauge fields have been widely studied [22-35]. The usual way to localize the U(1) gauge field is adding couplings [22-25, 27-29, 31, 34]. For these mechanisms, some questions have been raised in ref. [35]. For example, some additional couplings are included in refs. [23-25, 29], but it is not clear what the meaning of the additional couplings is. Do such mechanisms work for other braneworld models? Therefore, refs. [26, 30, 32, 33, 35, 36] proposed geometrical coupling mechanisms by introducing the coupling terms $R A_{M} A^{M}$ and $R_{M N} A^{M} A^{N}$, and found that the unique condition for localization is that the bulk is asymptotically AdS [35]. For geometrical coupling mechanisms, we still have some questions. What do these coupling terms mean? These coupling terms have effect on the four-dimensional effective action. Whether the four-dimensional effective theory coincides with observations, especially in a strong gravity region? In view of all this, we will consider the minimal coupling between the $\mathrm{U}(1)$ gauge field and gravity in this paper.

In ref. [35], the authors found that the localization condition for the free $\mathrm{U}(1)$ gauge field in an asymptotically AdS spacetime is $(D-4)>d$, where $D$ is the number of the bulk dimensions and $d$ is the number of infinite extra dimensions. Note that, for a fixed $d$, the condition $(D-4)>d$ can always be satisfied by introducing compact extra dimensions. In this case, the matter fields need to be localized not on the 3-brane but on a $(D-d)$-dimensional submanifold containing these compact dimensions. For example, six-dimensional models with an infinite extra dimension and a compact one satisfy this condition.

The types of six-dimensional braneworld models can be classified by the geometry of the extra-dimensional space (the transverse space) which may

- be two infinitely large dimensions [37-40],

- be a 2 -sphere [41], a football shape [42], or an apple shape [43],

- be a torus $[44,45]$,

- include a conical deficit [46-53], and

- be a cylinder [54-57].

The models with a cylinder-like transverse space whose geometry satisfies the localization condition of free vector fields are studied in refs. [54-57]. They suggest that the matter fields are localized on a 4 -brane denoted by 5-diemnsional manifold $\mathcal{M}_{4} \times \mathcal{S}_{1}$, while the 3-brane corresponds to $\mathcal{M}_{4}$.

The consistency conditions of localization of the six-dimensional free $\mathrm{U}(1)$ gauge field have been investigated in refs. [58-60], which must be satisfied for a six-dimensional braneworld model. The geometry in ref. [54] satisfies the localization condition of the free vector field but is not generated by dynamical fields. The aim of this paper is to 
propose a braneworld model which satisfies both the consistency conditions [58] and the localization conditions [35] of the free $\mathrm{U}(1)$ gauge field. We expect that our model has the following properties.

- It is a thick brane model which includes a compact extra dimension and an infinite one.

- The brane is generated by a dynamical field.

- The spacetime is stable.

- The Newtonian potential can be recovered.

- To be consistent with observations, the compact extra dimension should be sufficiently small.

This paper is organized as follows. In section 2, we introduce the setup of our model. In section 3, we present a new brane solution. In section 4 , the tensor mode of gravitational perturbations is studied. In section 5 , the localization of boson fields is explored. The last section 6 is devoted to conclusions and outlook.

\section{Setup}

We consider a six-dimensional braneworld model constructed by a real scalar field $\phi$, and start with the action

$$
S=\frac{M_{*}^{4}}{2} \int d^{6} x \sqrt{-g}\left(R+\mathcal{L}_{\mathrm{m}}\right),
$$

where $g=\operatorname{det}\left(g_{M N}\right)$ with $M$ and $N$ denoting six-dimensional spacetime indices. Here $M_{*}$ is the six-dimensional fundamental energy scale. We use the unit $M_{*}=c=\hbar=1$ in the following. The Lagrangian of the background scalar field is

$$
\mathcal{L}_{\mathrm{m}}=-\frac{1}{2} g^{M N} \partial_{M} \phi \partial_{N} \phi-V_{\Lambda}(\phi),
$$

where

$$
V_{\Lambda}(\phi)=V(\phi)+\Lambda
$$

Here we write the six-dimensional cosmological constant $\Lambda$ independently. From the Lagrangian (2.2), the energy-momentum tensor is

$$
T_{M N} \equiv-\frac{2}{\sqrt{-g}} \frac{\delta\left(\sqrt{-g} \mathcal{L}_{\mathrm{m}}\right)}{\delta g^{M N}}=\partial_{M} \phi \partial_{N} \phi+g_{M N} \mathcal{L}_{\mathrm{m}}
$$

The variation of the action (2.1) with respect to the metric $g_{M N}$ yields the field equation

$$
R_{M N}-\frac{1}{2} g_{M N} R=T_{M N},
$$

and the equation of motion for the scalar field $\phi$ is

$$
\square^{(6)} \phi=\frac{\partial V_{\Lambda}}{\partial \phi},
$$

where $\square^{(6)}=g^{M N} \nabla_{M} \nabla_{N}$ is the six-dimensional d'Alembert operator. 
In this work, we are interested in a six-dimensional spacetime $\mathcal{M}_{4} \times \mathcal{R}_{1} \times \mathcal{S}_{1}$, where $\mathcal{M}_{4}$ is a four-dimensional Minkowski manifold and $\mathcal{R}_{1} \times \mathcal{S}_{1}$ is a transverse manifold. The metric ansatz can be written as

$$
d s^{2}=a^{2}(y) \eta_{\mu \nu} d x^{\mu} d x^{\nu}+d y^{2}+b^{2}(y) R_{0}^{2} d \theta^{2},
$$

where $y \in(-\infty, \infty)$ denotes the infinite extra dimension and $\theta \in[0,2 \pi)$ denotes the compact extra dimension with radius $R_{0}$. Similar to the KK theory, we assume that $R_{0}$ is sufficiently small, so that the experimental energy scale $(\mathrm{TeV})$ does not allow us to access it. With the coordinate transformation $\Theta=R_{0} \theta$, the metric becomes

$$
d s^{2}=a^{2}(y) \eta_{\mu \nu} d x^{\mu} d x^{\nu}+d y^{2}+b^{2}(y) d \Theta^{2} .
$$

Here the warp factors $a(y)$ and $b(y)$ are only dependent on the coordinate $y$, and $\eta_{\mu \nu}=$ $\operatorname{diag}(-1,1,1,1)$ is the Minkowski metric. With these assumptions, the field equation (2.5) reduces to

$$
\begin{array}{ll}
(\mu, \nu): & \frac{3 a^{\prime \prime}}{a}+\frac{3 a^{\prime} b^{\prime}}{a b}+\frac{3 a^{\prime 2}}{a^{2}}+\frac{b^{\prime \prime}}{b}=-\frac{1}{2} \phi^{2}-V_{\Lambda}(\phi), \\
(y, y): & \frac{4 a^{\prime} b^{\prime}}{a b}+\frac{6 a^{\prime 2}}{a^{2}}=\frac{1}{2} \phi^{\prime 2}-V_{\Lambda}(\phi), \\
(\theta, \theta): & \frac{4 a^{\prime \prime}}{a}+\frac{6 a^{\prime 2}}{a^{2}}=-\frac{1}{2} \phi^{\prime 2}-V_{\Lambda}(\phi),
\end{array}
$$

and the equation of motion for the scalar field (2.6) can be rewritten as

$$
\phi^{\prime \prime}+\left(4 \frac{a^{\prime}}{a}+\frac{b^{\prime}}{b}\right) \phi^{\prime}-\frac{\partial V_{\Lambda}}{\partial \phi}=0,
$$

where the prime represents the derivative with respect to $y$. Note that only three equations in eqs. (2.9) and (2.10) are independent, while four functions need to be solved. This allows us to make some reasonable assumptions with regard to the scalar field to guarantee our solution satisfying the conditions

$$
\left.a(y)\right|_{y=0}=1,\left.\quad a^{\prime}(y)\right|_{y=0}=0,\left.\quad b(y)\right|_{y=0} \neq 0,\left.\quad b^{\prime}(y)\right|_{y=0}=0 .
$$

In this situation, we start with eqs. (2.9) and (2.10) to obtain two analytical solutions in the following section.

\section{Two braneworld solutions in six-dimensional asymptotically AdS spacetime}

In this section, we look for solutions satisfying the above conditions (2.11).

\subsection{Solution 1}

We adopt the following assumption for the scalar potential $V_{\Lambda}(\phi)$ with

$$
\begin{aligned}
V(\phi) & =\frac{k^{2} v^{2}}{2}+\frac{5}{18} k^{2} v^{4}-\left(k^{2}+\frac{5 k^{2} v^{2}}{8}\right) \phi^{2}+\left(\frac{5 k^{2}}{12}+\frac{k^{2}}{2 v^{2}}\right) \phi^{4}-\frac{5 k^{2}}{72 v^{2}} \phi^{6}, \\
\Lambda & =-\frac{5}{18} k^{2} v^{4} .
\end{aligned}
$$


Then, from the Einstein equation (2.9), the scalar field and the warp factors are obtained as

$$
\begin{aligned}
& \phi(y)=v \tanh (k y), \\
& a(y)=b(y)=\mathrm{e}^{-\frac{1}{24} v^{2} \tanh ^{2}(k y)} \operatorname{sech}^{\frac{v^{2}}{6}}(k y) .
\end{aligned}
$$

Here $v$ is a dimensionless parameter and $k$ is a fundamental energy scale with dimension $[k]=L^{-1} \cdot 1 / k$ stands for the thickness of the brane.

\subsection{Solution 2}

Another solution can be found if the scalar potential $V(\phi)$ and the cosmological constant $\Lambda$ are assumed as

$$
\begin{aligned}
V(\phi) & =\left(\frac{k^{2}}{2}+\frac{5 k^{2} v^{2}}{24}\right) \phi^{2}-\left(\frac{5 k^{2}}{24}+\frac{k^{2}}{2 v^{2}}\right) \phi^{4}+\frac{5 k^{2}}{72 v^{2}} \phi^{6}, \\
\Lambda & =-\frac{5 k^{2} v^{4}}{72} .
\end{aligned}
$$

Then, the scalar field and the warp factors are obtained as

$$
\begin{aligned}
& \phi(y)=v \operatorname{sech}(k y), \\
& a(y)=b(y)=\mathrm{e}^{\frac{1}{24} v^{2} \tanh ^{2}(k y)} \operatorname{sech}^{\frac{v^{2}}{12}}(k y) .
\end{aligned}
$$

Unless otherwise specified, the following discussion in this paper is based on the solution 2. For both solutions, the line element has the following form:

$$
d s^{2}=a^{2}(y) \eta_{\mu \nu} d x^{\mu} d x^{\nu}+d y^{2}+a^{2}(y) d \Theta^{2} .
$$

The main difference between solution 1 and solution 2 is that the brane does not split for solution 1 while splitting for solution 2 .

The scalar potential $V(\phi)$ (3.5) and the scalar field $\phi(3.7)$ are shown in figure 1. Noticing that when $y \rightarrow \pm \infty$, the scalar field $\phi(y) \rightarrow 0$ and the scalar potential $V(\phi) \rightarrow 0$, the contribution to gravity only comes from the cosmological constant. This means that the bulk is an asymptotically $\operatorname{AdS}_{6}$ spacetime. When $v<\frac{6}{\sqrt{5}}$, the AdS vacuum is a false one, and the spacetime may be unstable by considering quantum tunneling. While it corresponds to a true vacuum when $v>\frac{6}{\sqrt{5}}$. When $v=\frac{6}{\sqrt{5}}$, there are three degenerate vacua and the vacuum expectation values are $\left\{0, \pm \frac{6 \sqrt{2}}{\sqrt{5}}\right\}$.

For an observer with time-like 6-veiocity satisfying $g_{M N} U^{M} U^{N}=-1$, the energy density of the background scalar field reads

$$
\rho=T_{M N} U^{M} U^{N},
$$

where the cosmological constant is not included. For a static observer, the energy density $\rho=T_{0}^{0}$ is shown in figure 2 .

The volcano-shaped energy density in figure 2 shows that the brane has inner structures. By comparing $\rho_{0} \equiv \rho(0)$ and $\rho_{\max } \equiv \operatorname{Max}[\rho(y)]$, we can define

$$
\lambda(v)=\left(\rho_{\max }-\rho_{0}\right) / \rho_{\max },
$$




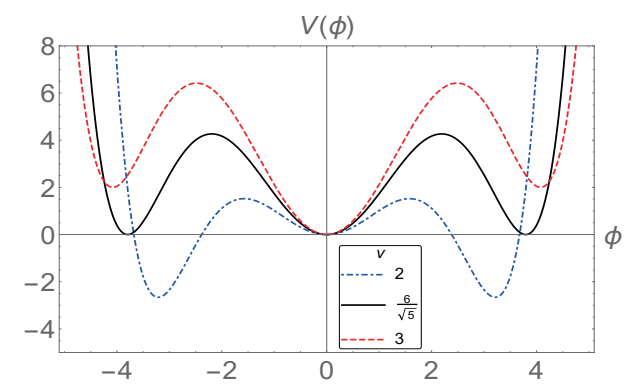

(a) Scalar potential

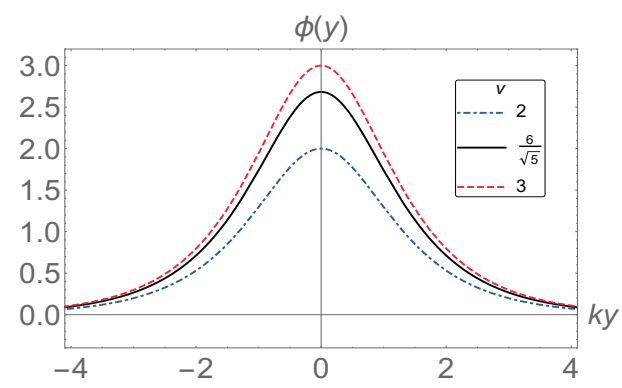

(b) Scalar field

Figure 1. Plots of the scalar potential (3.5) and background scalar field (3.7). The scalar field $\phi(y) \rightarrow 0$ as $y \rightarrow \pm \infty$, which corresponds to the local minimum $V(0)$ of the scalar potential.

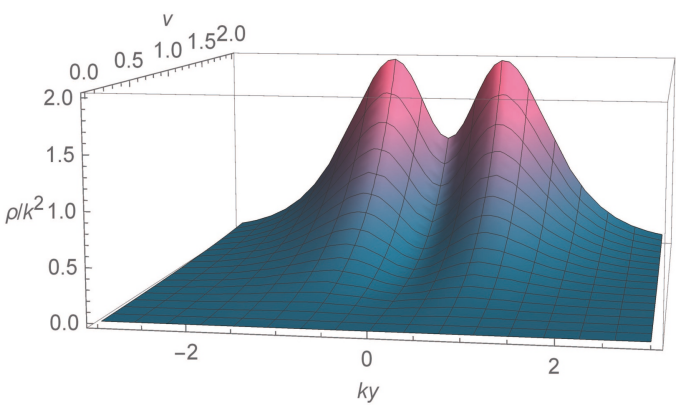

(a)

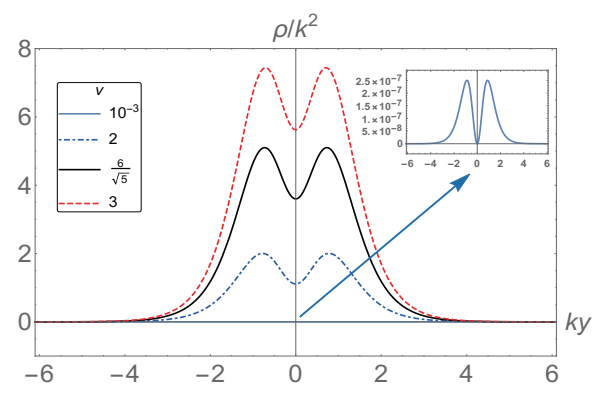

(b)

Figure 2. The energy density of the background scalar field. Figure 2(a) shows the change of the energy density with respect to the parameter $v$, and figure 2(b) shows that the brane splits into two separated sub-branes as $v \rightarrow 0$.

which represents the relative degree of splitting of the brane. Note that this scheme is only suitable for studying the degree of brane splitting, it can not well reflect the degree of separation between two sub-branes. When $\lambda(v)>1 / 2$, we can deem that the two sub-branes are separated. In this case, for two symmetric sub-branes, we can define the full width at half maximum (FWHM) for each sub-brane. Then, we present the following method to measure the degree of separation between the two sub-branes as

$$
\tilde{\lambda}(v)=\frac{\text { Interval between two sub-branes }}{\text { FWHM for each sub-brane }} \text {. }
$$

See figure 3 for the schematic diagram of the above description.

The above definition is to facilitate the generalization to the case of multiple subbranes. Here we just use the first scheme for our model. We find that $\lambda(v) \rightarrow 1$ as $v \rightarrow 0$ (as shown in figure 2(b)), and the degree of splitting $\lambda(v)$ decreases with $v$ (as shown in figure 2(a)). The brane is always splitting for any value of $v$, and this result can be obtained from $\rho^{\prime \prime}(y)>0$ at $y=0$.

We show the warp factor (3.8) in figure 4(a) and also draw the profile of the extra dimensions described by the line element $d s_{\text {extra }}^{2}=d y^{2}+b^{2}(y) R_{0}^{2} d \theta^{2}$ in figure 5 . The transverse space contains a noncompact dimension and a compact one. Comparing with a 


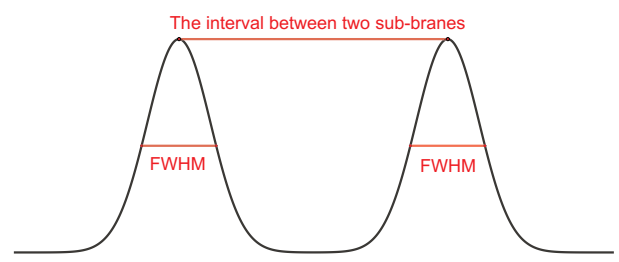

Figure 3. The schematic diagram of the degree of separation between two sub-branes.

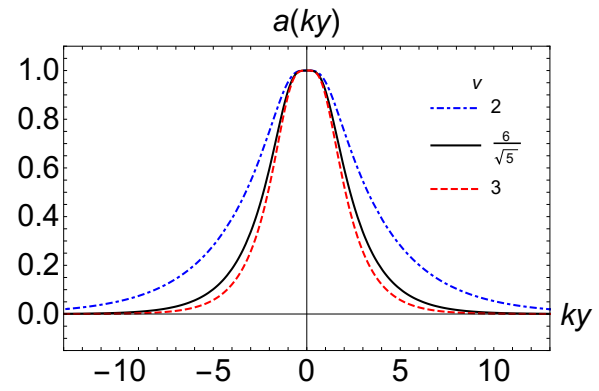

(a) Warp factor

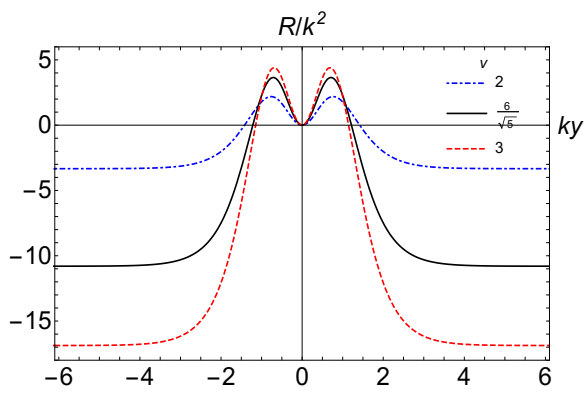

(b) Scalar curvature

Figure 4. Plots of the warp factor (3.8) and the corresponding scalar curvature. The spacetime is asymptotically $\mathrm{AdS}_{6}$ at $y \rightarrow \pm \infty$ and Minkowski at $y=0$.

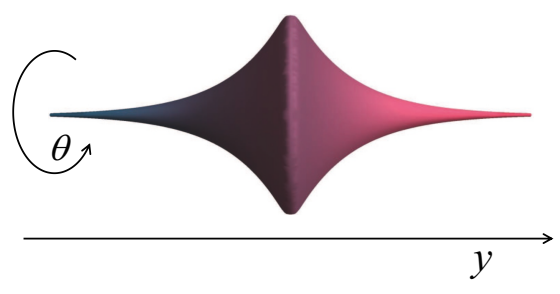

Figure 5. The profile of the extra dimensions described by the line element $d s_{\text {extra }}^{2}=d y^{2}+$ $b^{2}(y) R_{0}^{2} d \theta^{2}$. The parameter $v$ is set to 1 . The compact radius decreases with $|y|$, which makes the compact extra dimension difficult to detect. With the warp factor $b(y)(3.8)$, we find that the area of this two-dimensional surface is finite. This means that although there is an infinitely large transverse space, the volume of extra dimensions can still be finite. If there is a smooth finite function on the extra-dimensional manifold, its integral with respect to the extra dimensions will be finite.

noncompact five-dimensional configuration, the compact sixth dimension will result in an expected effect on localization as shown in section 5 .

\section{Linear tensor perturbations and localization of the graviton}

It is well known that the perturbations of the background can be decomposed into three kinds under the four-dimensional Lorentz transformation: the transverse-traceless (TT) tensor mode, the transverse vector modes, and the scalar modes, namely the scalar-vectortensor decomposition. The three kinds of modes decouple from each other in linear or- 
der [61-65]. So each kind of modes can be investigated independently. In this work, we will only investigate the TT tensor mode, which can be written as

$$
\delta g_{M N}=a^{2}(y)\left(\begin{array}{cc}
h_{\mu \nu}\left(x^{\sigma}, y, \Theta\right) & 0_{4 \times 2} \\
0_{2 \times 4} & 0_{2 \times 2}
\end{array}\right) .
$$

Without considering the TT condition, we show the first-order perturbations of the Ricci tensor

$$
\begin{aligned}
\delta R_{\mu \nu}= & \frac{1}{2} \partial_{\nu} \partial_{\lambda} h_{\mu}^{\lambda}+\frac{1}{2} \partial_{\mu} \partial_{\lambda} h_{\nu}^{\lambda}-\frac{1}{2} \square^{(4)} h_{\mu \nu}-\frac{1}{2} \partial_{\mu} \partial_{\nu} h \\
& -a a^{\prime \prime} h_{\mu \nu}-3 a^{\prime 2} h_{\mu \nu}-2 a a^{\prime} h_{\mu \nu}^{\prime}-\frac{1}{2} a^{2} h_{\mu \nu}^{\prime \prime}-\frac{1}{2} a a^{\prime} h^{\prime} \eta_{\mu \nu} \\
& -\frac{1}{2} \frac{a^{2}}{b^{2}} \partial_{\Theta}^{2} h_{\mu \nu}-\frac{1}{2} \frac{a^{2} b^{\prime}}{b} h_{\mu \nu}^{\prime}-\frac{a a^{\prime} b^{\prime}}{b} h_{\mu \nu}, \\
\delta R_{\mu y}= & -\frac{1}{2} \partial_{\mu} h^{\prime}+\frac{1}{2} \partial^{\lambda} h_{\mu \lambda}^{\prime}, \\
\delta R_{\mu \Theta}= & \frac{1}{2} \partial_{\Theta} \partial_{\lambda} h_{\mu}^{\lambda}-\frac{1}{2} \partial_{\Theta} \partial_{\mu} h \\
\delta R_{y y}= & -\frac{1}{2} h^{\prime \prime}-\frac{a^{\prime}}{a} h^{\prime}, \\
\delta R_{y \Theta}= & -\frac{1}{2} \partial_{\Theta} h^{\prime}+\frac{1}{2}\left(\frac{b^{\prime}}{b}-\frac{a^{\prime}}{a}\right) \partial_{\Theta} h \\
\delta R_{\Theta \Theta}= & -\frac{1}{2} \partial_{\Theta}^{2} h-\frac{1}{2} b b^{\prime} h^{\prime},
\end{aligned}
$$

and the first-order perturbation of the scalar curvature

$$
\delta R=\frac{1}{a^{2}} \partial_{\nu} \partial_{\lambda} h^{\nu \lambda}-\frac{1}{a^{2}} \square^{(4)} h-\frac{1}{b^{2}} \partial_{\Theta}^{2} h-h^{\prime \prime}-\left(5 \frac{a^{\prime}}{a}+\frac{b^{\prime}}{b}\right) h^{\prime},
$$

where $\square^{(4)}=\eta^{\mu \nu} \partial_{\mu} \partial_{\nu}$ is the four-dimensional d'Alembert operator and $h=\eta^{\mu \nu} h_{\mu \nu}$. Taking into account the TT condition $h=0=\partial^{\mu} h_{\mu \nu}$, the equation for the perturbations $h_{\mu \nu}$ reduces to

$$
\frac{1}{a^{2}} \square^{(4)} h_{\mu \nu}+\frac{1}{b^{2}} \partial_{\Theta}^{2} h_{\mu \nu}+h_{\mu \nu}^{\prime \prime}+\left(4 \frac{a^{\prime}}{a}+\frac{b^{\prime}}{b}\right) h_{\mu \nu}^{\prime}=0 .
$$

This equation can also be derived by varying the gravitational action including the quadratic terms of the tensor perturbations. More generally, the above equation can be written as

$$
\square^{(6)} h_{\mu \nu}=0 .
$$

The corresponding action can be written as

$$
S_{2} \sim \int d^{6} x \sqrt{-g} g^{M N} \nabla_{M} h_{\mu \nu} \nabla_{N} h^{\mu \nu} .
$$

With the coordinate transformation

$$
d z=\frac{d y}{a(y)}
$$


we can rewrite the perturbation equation (4.4) as

$$
\square^{(4)} h_{\mu \nu}+\frac{a^{2}}{b^{2}} \partial_{\Theta}{ }^{2} h_{\mu \nu}+\partial_{z}^{2} h_{\mu \nu}+\left(\frac{\partial_{z} b}{b}+3 \frac{\partial_{z} a}{a}\right) \partial_{z} h_{\mu \nu}=0 .
$$

Performing the KK decomposition

$$
h_{\mu \nu}\left(x^{\sigma}, z, \Theta\right)=\sum_{m, n} \hat{h}_{\mu \nu}^{(m)}\left(x^{\sigma}\right) \varphi_{(m, n)}(z) e^{i l_{n} \Theta}
$$

where $e^{i l_{n} \Theta}$ is the compact dimensional part, we obtain the Klein-Gordon (KG) equation for the four-dimensional part $\hat{h}_{\mu \nu}^{(m)}\left(x^{\sigma}\right)$ :

$$
\left(\square^{(4)}-m^{2}\right) \hat{h}_{\mu \nu}^{(m)}\left(x^{\sigma}\right)=0
$$

and the equation for the noncompact extra-dimensional part $\varphi_{m n}(z)$ :

$$
\partial_{z}^{2} \varphi_{(m, n)}+\left(\frac{\partial_{z} b}{b}+3 \frac{\partial_{z} a}{a}\right) \partial_{z} \varphi_{(m, n)}+\left(m^{2}-\frac{a^{2}}{b^{2}} l_{n}^{2}\right) \varphi_{(m, n)}=0 .
$$

Here $m$ can be interpreted as the effective mass of the KK graviton, and the azimuthal number $l_{n}=n / R_{0}$, where $n$ is an integer due to the periodic boundary condition.

With the transformation

$$
\varphi_{(m, n)}=a^{-\frac{3}{2}} b^{-\frac{1}{2}} \tilde{\varphi}_{(m, n)},
$$

eq. (4.11) can be rewritten as a Schrödinger-like equation

$$
\left[-\partial_{z}^{2}+U_{2}(z)\right] \tilde{\varphi}_{(m, n)}(z)=\left(m^{2}-\frac{a^{2}}{b^{2}} l_{n}^{2}\right) \tilde{\varphi}_{(m, n)}(z)
$$

where the effective potential $U_{2}(z)$ has the form

$$
U_{2}(z)=\frac{3}{4} \frac{\left(\partial_{z} a\right)^{2}}{a^{2}}-\frac{1}{4} \frac{\left(\partial_{z} b\right)^{2}}{b^{2}}+\frac{6}{4} \frac{\partial_{z} a}{a} \frac{\partial_{z} b}{b}+\frac{3}{2} \frac{\partial_{z}^{2} a}{a}+\frac{1}{2} \frac{\partial_{z}^{2} b}{b}
$$

With the braneworld solution (3.8), the effective potential reduces to

$$
U_{2}(z)=2 \frac{\left(\partial_{z} a\right)^{2}}{a^{2}}+2 \frac{\partial_{z}^{2} a}{a} .
$$

As shown in figure 6 (a), we can find that $U_{2}(z) \rightarrow 0$ when $z \rightarrow \infty$. This means that the KK modes with $m^{2}-l_{n}^{2}>0$ are free states.

Eq. (4.13) can be factorized as

$$
\left(-\partial_{z}-2 \frac{\partial_{z} a}{a}\right)\left(\partial_{z}-2 \frac{\partial_{z} a}{a}\right) \tilde{\varphi}_{(m, n)}(z)=\left(m^{2}-l_{n}^{2}\right) \tilde{\varphi}_{(m, n)}(z) .
$$

We give a simple discussion for several cases. For convenience, we adopt $m_{n}=\left|l_{n}\right|=|n| / R_{0}$ to label some special values of $m$. 


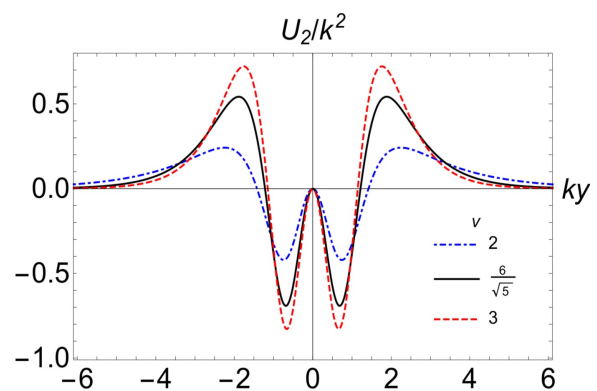

(a) The effective potential of the graviton

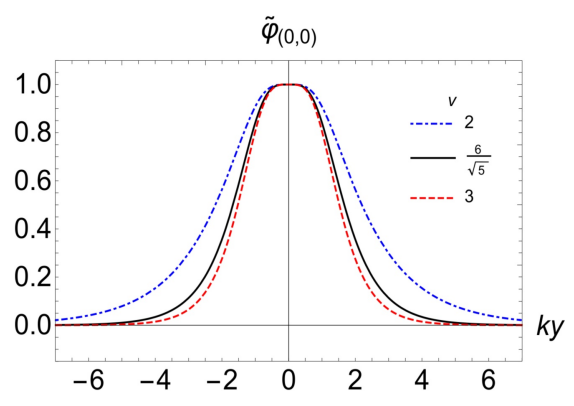

(b) The wave function of the ground state with the eigenvalue $m_{n}^{2}-l_{n}^{2}=0$

Figure 6. The effective potential and the wave function of the ground state of eq. (4.13).

- It is clear that eq. (4.16) has the form as $A^{\dagger} A \tilde{\varphi}_{(m, n)}(z)=\left(m^{2}-l_{n}^{2}\right) \tilde{\varphi}_{(m, n)}(z)$, where $A=\partial_{z}-2\left(\partial_{z} a\right) / a$. According to the Sturm-Liouville theorem, the lowest energy state of the Schrödinger-like equation has no zero point in its domain of definition. The eigenvalue corresponding to the lowest energy state is $m_{n}^{2}-l_{n}^{2}=0$. This indicates that eigenvalues are nonnegative $\left(m^{2}-l_{n}^{2} \geqslant 0\right)$. Therefore, the system is spatially stable under the tensor perturbations in the linear order.

- To avoid tachyons and to ensure that the system is time-stable, $m^{2} \geqslant 0$ is required from the KG equation (4.10).

- The wave function of the ground state $\left(m_{n}^{2}-l_{n}^{2}=0\right)$ for eq. (4.16) is $\tilde{\varphi}_{\left(m_{n}, n\right)}(z)=$ $C_{1} a^{2}(z)$ (we have ruled out another linearly independent particular solution that does not satisfy the localization condition), where $C_{1}$ is an integration constant.

Due to $m^{2}-l_{n}^{2} \geqslant 0$, the state with $m_{0}=0$ only has the s-wave $\left(l_{0}=0\right)$ mode. To obtain the four-dimensional massless graviton, the massless s-wave $\left(m_{0}=l_{0}=0\right)$ mode $\tilde{\varphi}_{(0,0)}(z)$ should be normalizable. The normalization condition for $\tilde{\varphi}_{(0,0)}(z)$ is

$$
\int_{-\infty}^{+\infty}\left|\tilde{\varphi}_{(0,0)}(z)\right|^{2} d z=\int_{-\infty}^{+\infty}\left|\tilde{\varphi}_{(0,0)}(y)\right|^{2} a^{-1}(y) d y=C_{1}^{2} \int_{-\infty}^{+\infty} a^{3}(y) d y=1 .
$$

Here $C_{1}$ is in fact a normalization constant. For the warp factor (3.8), we have

$$
\int_{-\infty}^{+\infty} d y a^{p}(y)<\infty, \text { when } p>0
$$

In this six-dimensional braneworld model, all the ground states $\tilde{\varphi}_{\left(m_{n}, n\right)}$ with $m_{n}=\left|l_{n}\right|=$ $|n| / R_{0}$ can be localized on the brane. This means that there is a discrete spectrum with the effective mass $m_{n}=|n| / R_{0}$ in the four-dimensional effective theory.

The four-dimensional effective gravity comes from the contribution of the massless s-wave mode $\left(m_{0}=l_{0}=0\right)$, the discrete modes of localized four-dimensional massive gravitons $\left(m_{n}=\left|l_{n}\right|=|n| / R_{0}\right)$, and the continuous modes $\left(m^{2}-l_{n}^{2}>0\right)$. Noticing that the massless s-wave mode is localized on the brane, it will lead to the Newtonian potential. Moreover the other KK modes will lead to the corrections to the Newtonian potential. 
When the thickness of the brane is small compared with the length corresponding to the fundamental energy scale $M_{*}$, the gravitational potential between two particles with mass $M_{1}$ and $M_{2}$ located at $y=0$ can be written as

$$
U(r) \sim \sum_{n=-\infty}^{\infty} G_{n} \frac{M_{1} M_{2} e^{-m_{n} r}}{r}+\frac{1}{2 \pi R_{0} M_{*}^{4}} \sum_{n=-\infty}^{\infty} \int_{\left|l_{n}\right|}^{\infty} d m \frac{M_{1} M_{2} e^{-m r}}{r}\left|\bar{\varphi}_{(m, n)}(0)\right|^{2}
$$

Here the effective gravitational constant can be calculated by the relative probability density of the KK modes at $y=0$ as

$$
G_{n} \sim \frac{1}{2 \pi R_{0} M_{*}^{4}}\left|\bar{\varphi}_{\left(m_{n}, n\right)}(0)\right|^{2}=\frac{1}{2 \pi R_{0} M_{*}^{4}} \frac{\left|\tilde{\varphi}_{\left(m_{n}, n\right)}(0)\right|^{2}}{\left\langle\tilde{\varphi}_{\left(m_{n}, n\right)} \mid \tilde{\varphi}_{\left(m_{n}, n\right)}\right\rangle},
$$

where $\bar{\varphi}_{\left(m_{n}, n\right)}(z)=\tilde{\varphi}_{\left(m_{n}, n\right)}(z) / \sqrt{\left\langle\tilde{\varphi}_{\left(m_{n}, n\right)} \mid \tilde{\varphi}_{\left(m_{n}, n\right)}\right\rangle}$ are the normalized wave functions with $m_{n}=\left|l_{n}\right|=|n| / R_{0}$. Note that we have $G_{n}=G_{0}=G_{\mathrm{N}}$ for any $n$, where $G_{\mathrm{N}}$ is the Newtonian constant. The contribution of the continuous states comes from all possible KK modes with $m^{2}-l_{n}^{2}>0$. The wavefunctions $\bar{\varphi}_{m}(y)$ of the continuous states are normalized as plane waves, i.e. to unity over a period at $|y| \rightarrow \infty$ [11]. The $d$-dimensional plane wave continuum density of states $m^{d-1}$ has been considered here [11] (the number of the infinite extra dimensions $d=1$ in this model).

Recall that we have decomposed the solution to the equation of perturbations as a sum over Kaluza-Klein modes. For bound states, the mass gap is $1 / R_{0}$. Noticing that

$$
\sum_{n=-\infty}^{\infty} G_{n} \frac{M_{1} M_{2} e^{-m_{n} r}}{r}=G_{\mathrm{N}} \frac{M_{1} M_{2}}{r}\left(1+\frac{2}{e^{r / R_{0}}-1}\right)
$$

it is easy to obtain the asymptotic behavior at short $\left(r \ll R_{0}\right)$ and long $\left(r \gg R_{0}\right)$ distances:

$$
\sum_{n=-\infty}^{\infty} G_{n} \frac{M_{1} M_{2} e^{-m_{n} r}}{r} \sim\left\{\begin{array}{l}
\frac{R_{0}}{r^{2}}, \text { for } r \ll R_{0}, \\
\frac{1}{r}, \text { for } r \gg R_{0} .
\end{array}\right.
$$

At distances shorter than the compactification radius $R_{0}$, Newtonian potential will be modified [1]. The experimental tests of the gravitational force are usually parametrized by the modified potential [66-68]

$$
U(r) \sim G \frac{m_{1} m_{2}}{r}[1+\alpha \exp (-r / \lambda)]
$$

From eq. (4.19), considering the contribution of the KK modes with $m_{0}=0$ and $m_{1}=1 / R_{0}$, it is easy to get the parameters as $\alpha=2, \lambda=R_{0}$. The inverse-square law has been experimentally verified at sub-millimeter distances [66-72], which imposes constraints on our model that $R_{0}$ should be less than sub-millimeter.

From eq. (4.22), it has been shown that the massive KK modes are suppressed at long distances, or equivalently at low energies. Hence, it makes sense to write down an action which only includes the massless mode with $n=0$. Plugging the massless mode $\bar{\varphi}_{(0,0)}(z)$ into the action (4.6), we obtain the low energy effective action

$$
S_{\mathrm{eff}}=M_{*}^{4} \pi R_{0} \int \bar{\varphi}_{(0,0)}^{2}(z) d z \int d^{4} x\left[-\frac{1}{4} \eta^{\mu \nu} \partial_{\mu} \hat{h}_{\alpha \beta}^{(0)}\left(x^{\sigma}\right) \partial_{\nu} \hat{h}^{(0) \alpha \beta}\left(x^{\sigma}\right)\right] .
$$


It can also be viewed as a low energy approximation of general relativity in four-dimensional spacetime and the Newtonian potential can be obtained by this action.

\section{Localization of scalar fields and vector fields}

In this section, we investigate the localization of the bulk massless scalar field and the free $\mathrm{U}(1)$ gauge field, and expect to obtain a four-dimensional effective description.

\subsection{Scalar fields}

The action for a massless scalar field in six-dimensional spacetime reads

$$
S_{0}=-\frac{1}{2} \int d^{6} x \sqrt{-g} g^{M N} \partial_{M} \Phi^{*} \partial_{N} \Phi
$$

and the corresponding equation of motion is

$$
\square^{(6)} \Phi=0 .
$$

With the metric (3.9) and performing the KK decomposition

$$
\Phi\left(x^{\sigma}, y, \Theta\right)=\sum_{m, n} \phi^{(m, n)}\left(x^{\sigma}\right) \varphi_{(m, n)}(y) e^{i l_{n} \Theta}
$$

the action (5.1) can be written as (considering the different KK modes are orthogonal to each other)

$$
\begin{aligned}
S_{0}=\sum_{m, n}-\frac{1}{2}\left[I_{1(m, n)} \int d^{4} x \eta^{\mu \nu} \partial_{\mu} \phi^{*(m, n)}\left(x^{\sigma}\right) \partial_{\nu} \phi^{(m, n)}\left(x^{\sigma}\right)\right. \\
\\
\left.+I_{2(m, n)} \int d^{4} x \phi^{*(m, n)}\left(x^{\sigma}\right) \phi^{(m, n)}\left(x^{\sigma}\right)\right],
\end{aligned}
$$

where

$$
\begin{aligned}
& I_{1(m, n)}=2 \pi \int d y a^{3}(y) \varphi_{(m, n)}^{*}(y) \varphi_{(m, n)}(y) \\
& I_{2(m, n)}=2 \pi \int d y\left[a^{5}(y) \partial_{y} \varphi_{(m, n)}^{*}(y) \partial_{y} \varphi_{(m, n)}(y)+a^{3}(y) l_{n}^{2} \varphi_{(m, n)}^{*}(y) \varphi_{(m, n)}(y)\right] .
\end{aligned}
$$

It is easy to see that $I_{2(m, n)}$ corresponds to the mass parameter of a scalar field $\phi^{(m, n)}\left(x^{\sigma}\right)$ in the four-dimensional effective theory. The localization condition of the zero mode requires

$$
I_{1(0,0)}<\infty
$$

The solution of the zero mode $\left(I_{2(0,0)}=0\right)$ is $\varphi_{(0,0)}(y)=C_{1}$ (satisfying the field equation and the localization condition). This means that the scalar field is homogeneously distributed in the transverse space. But it is still localized. The reason is that the spacetime is curved and the warp factor contributes to the integral. The following discussion may help us to understand this. The effect of the curved spacetime can be described equivalently in the following way. 
With the coordinate transformation (4.7), we can rewrite line element (3.9) with conformal coordinates

$$
d s^{2}=a^{2}(z)\left(\eta_{\mu \nu} d x^{\mu} d x^{\nu}+d z^{2}+d \Theta^{2}\right) .
$$

Considering the following field transformation

$$
\varphi_{(m, n)}=a^{-2}(z) \tilde{\varphi}_{(m, n)},
$$

we have

$$
\begin{aligned}
I_{1(m, n)}= & 2 \pi \int d y a^{3}(y) \varphi_{(m, n)}^{*}(y) \varphi_{(m, n)}(y)=2 \pi \int d z \tilde{\varphi}_{(m, n)}^{*}(z) \tilde{\varphi}_{(m, n)}(z), \\
I_{2(m, n)}= & 2 \pi \int d y\left[a^{5}(y) \partial_{y} \varphi_{(m, n)}^{*}(y) \partial_{y} \varphi_{(m, n)}(y)+a^{3}(y) l_{n}^{2} \varphi_{(m, n)}^{*}(y) \varphi_{(m, n)}(y)\right] \\
= & 2 \pi \int d z a^{4}(z) \partial_{z}\left[a^{-2}(z) \tilde{\varphi}_{(m, n)}^{*}(z)\right] \partial_{z}\left[a^{-2}(z) \tilde{\varphi}_{(m, n)}(z)\right] \\
& +2 \pi l_{n}^{2} \int d z a^{4}(z) \tilde{\varphi}_{(m, n)}^{*}(z) \tilde{\varphi}_{(m, n)}(z) .
\end{aligned}
$$

From eq. (5.10), we notice that the warp factor $a(z)$ has been absorbed in the field $\tilde{\varphi}(z)$. The effect of curved spacetime is equivalently represented with the transformation $\varphi_{(m, n)}(y) \rightarrow$ $\tilde{\varphi}_{(m, n)}(z)$ which satisfies the following Schrödinger-like equation

$$
\left[-\partial_{z}^{2}+U_{0}(z)\right] \tilde{\varphi}_{(m, n)}(z)=m^{2} \tilde{\varphi}_{(m, n)}(z),
$$

where the effective potential $U_{0}(z)$ has the form

$$
U_{0}(z)=2 \frac{\left(\partial_{z} a\right)^{2}}{a^{2}}+2 \frac{\partial_{z}^{2} a}{a} .
$$

The zero mode can be easily solved as

$$
\tilde{\varphi}_{(0,0)}(z)=C_{2} a^{2}(z)
$$

which corresponds to the solution $\varphi_{(0,0)}(y)=C_{2}$. It is important to emphasize that $\tilde{\varphi}_{(0,0)}(z)$ is a bound state.

Now we can easily consider the localization of the scalar field (the analytical method can also be applied to various matter fields) from two equivalent points of view.

- Physical coordinate view. The zero mode $\varphi_{(0,0)}(y)=C_{2}$ is homogeneously distributed in the transverse space and so it is localized anywhere. Notice that the spacetime is curved and there is a minimum coupling between gravity and a scalar field. The warp factor has a non-trivial contribution to the integral and the localization condition is satisfied as $I_{1(0,0)}=2 \pi \int d y a^{3}(y) \varphi_{(0,0)}^{*}(y) \varphi_{(0,0)}(y)<\infty$.

- Conformal coordinate view. Under the conformal coordinates and absorbing the conformal factor $a^{2}(z)$ into the field function $\tilde{\varphi}_{(m, n)}(z)$, the effect of curved spacetime is reflected by the effective potential (5.13) of the Schrödinger-like equation. The ground state wave function of the Schrödinger-like equation (5.12) is a bound state, i.e., $\tilde{\varphi}_{(0,0)}(z)=C_{2} a^{2}(z)$. This zero mode $\tilde{\varphi}_{(0,0)}(z)$ is localized near $z=0$ (see figure $7(\mathrm{~b})$ ). The localization condition is satisfied as $I_{1(0,0)}=2 \pi \int d z \tilde{\varphi}_{(0,0)}^{*}(z) \tilde{\varphi}_{(0,0)}(z)<\infty$. 


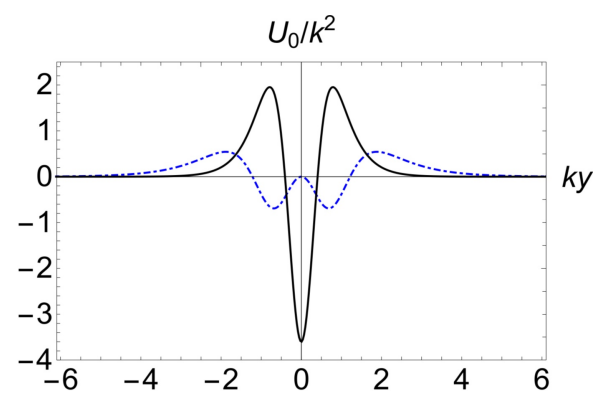

(a) The effective potential (5.13).

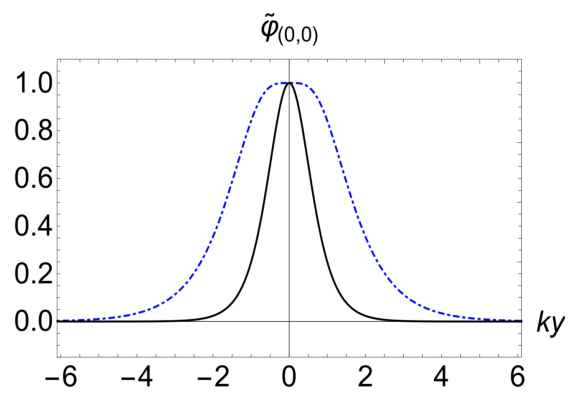

(b) The zero mode (5.14).

Figure 7. Localization of the scalar field under conformal flat spacetime view. The parameter $v=\frac{6}{\sqrt{5}}$. The blue dashed (black solid) line is corresponding to solution 2 (solution 1) where the brane is (is not) splitting.

These two statements are different descriptions of the same effect of gravity on the zero mode, but they are equivalent.

With the conformal coordinates view, figure $7(\mathrm{~b})$ shows clearly where the scalar field is localized when the brane splits. In our six-dimensional model, the scalar zero mode $\tilde{\varphi}_{(0,0)}(z)$ is localized near $z=0$. The zero mode does not split when the brane splits. By comparing solution 1 and solution 2, it can be found intuitively from figure $7(\mathrm{~b})$ that the scalar zero mode is localized over a wider area along the extra dimension when the brane splits.

\subsection{Vector fields}

In this subsection, we study the localization of the free $\mathrm{U}(1)$ gauge field following the method in ref. [58]. This method avoids the difficulty of solving the equation of motion of the vector field in a higher-dimensional curved spacetime. The effective action on the brane was derived by the KK decomposition of the vector field, and the consistency condition was obtained. Now we study whether these conditions are satisfied in our model.

We start with the action as

$$
S_{1}=-\frac{1}{4} \int d^{6} x \sqrt{-g} F^{M N} F_{M N}
$$

where

$$
F_{M N}=\partial_{M} A_{N}-\partial_{N} A_{M}
$$

is the field strength tensor.

Without loss of generality, we consider the following metric

$$
d s^{2}=a^{2}(z)\left(\hat{g}_{\mu \nu} d x^{\mu} d x^{\nu}+d z^{2}+d \Theta^{2}\right) .
$$

The quantities with hat "^" represent four-dimensional quantities whose indexes are raised by $\hat{g}^{\mu \nu}$. Such metric includes the case described by our solution. We use the notation $\partial^{\mu} \equiv \hat{g}^{\mu \nu} \partial_{\nu}$ in this section. This metric implies that the four-dimensional part and the 
extra-dimensional part can be separated through the general KK decomposition

$$
\begin{aligned}
& A_{\mu}=\sum_{m} \hat{X}_{\mu}^{(m)}\left(x^{\sigma}\right) W_{1}^{(m)}(z, \Theta) a^{-1} \\
& A_{z}=\sum_{m} \hat{\zeta}^{(m)}\left(x^{\sigma}\right) W_{2}^{(m)}(z, \Theta) a^{-1} \\
& A_{\Theta}=\sum_{m} \hat{\xi}^{(m)}\left(x^{\sigma}\right) W_{3}^{(m)}(z, \Theta) a^{-1} .
\end{aligned}
$$

We reduce the six-dimensional action to a four-dimensional effective one [58]

$$
\begin{aligned}
S_{1}= & -\frac{1}{4} \int d^{6} x \sqrt{-g} F^{M N} F_{M N} \\
= & -\frac{1}{4} \int d^{6} x \sqrt{-g}\left(F^{\mu_{1} \mu_{2}} F_{\mu_{1} \mu_{2}}+2 F^{\mu_{1} z} F_{\mu_{1} z}+2 F^{\mu_{1} \Theta} F_{\mu_{1} \Theta}+2 F^{\Theta z} F_{\Theta z}\right) \\
= & -\frac{1}{4} \sum_{m} \sum_{m^{\prime}} \int d^{4} x \sqrt{-\hat{g}}\left[I_{1}^{\left(m m^{\prime}\right)} \hat{F}_{\mu_{1} \mu_{2}}^{(m)} \hat{F}^{\mu_{1} \mu_{2}\left(m^{\prime}\right)}+\left(I_{2}^{\left(m m^{\prime}\right)}+I_{4}^{\left(m m^{\prime}\right)}\right) \hat{X}_{\mu_{1}}^{(m)} \hat{X}^{\mu_{1}\left(m^{\prime}\right)}\right. \\
& +I_{3}^{\left(m m^{\prime}\right)} \partial_{\mu_{1}} \hat{\zeta}^{(m)} \partial^{\mu_{1}} \hat{\zeta}^{\left(m^{\prime}\right)}-I_{6}^{\left(m m^{\prime}\right)}\left(\partial_{\mu_{1}} \hat{\zeta}^{(m)} \hat{X}^{\mu_{1}\left(m^{\prime}\right)}+\hat{X}_{\mu_{1}}^{(m)} \partial^{\mu_{1}} \hat{\zeta}^{\left(m^{\prime}\right)}\right) \\
& +I_{5}^{\left(m m^{\prime}\right)} \partial_{\mu_{1}} \hat{\xi}^{(m)} \partial^{\mu_{1}} \hat{\xi}^{\left(m^{\prime}\right)}-I_{8}^{\left(m m^{\prime}\right)}\left(\partial_{\mu_{1}} \hat{\xi}^{(m)} \hat{X}^{\mu_{1}\left(m^{\prime}\right)}+\hat{X}_{\mu_{1}}^{(m)} \partial^{\mu_{1}} \hat{\xi}^{\left(m^{\prime}\right)}\right) \\
& \left.+I_{7}^{\left(m m^{\prime}\right)} \hat{\zeta}^{(m)} \hat{\zeta}^{\left(m^{\prime}\right)}+I_{9}^{\left(m m^{\prime}\right)} \hat{\xi}^{(m)} \hat{\xi}^{\left(m^{\prime}\right)}-I_{10}^{\left(m m^{\prime}\right)}\left(\hat{\zeta}^{(m)} \hat{\xi}^{\left(m^{\prime}\right)}+\hat{\xi}^{(m)} \hat{\zeta}^{\left(m^{\prime}\right)}\right)\right]
\end{aligned}
$$

where $\hat{F}_{\mu \nu}^{(m)}=\partial_{\mu} \hat{X}_{\nu}^{(m)}-\partial_{\nu} \hat{X}_{\mu}^{(m)}$ and the constants are given by

$$
\begin{aligned}
I_{1}^{\left(m m^{\prime}\right)} & \equiv \int d \Theta d z W_{1}^{(m)} W_{1}^{\left(m^{\prime}\right)}, \\
I_{2}^{\left(m m^{\prime}\right)} & \equiv 2 \int d \Theta d z \partial_{\Theta}\left(W_{1}^{(m)} a^{-1}\right) \partial_{\Theta}\left(W_{1}^{\left(m^{\prime}\right)} a^{-1}\right) a^{2}, \\
I_{3}^{\left(m m^{\prime}\right)} & \equiv 2 \int d \Theta d z W_{2}^{(m)} W_{2}^{\left(m^{\prime}\right)}, \\
I_{4}^{\left(m m^{\prime}\right)} & \equiv 2 \int d \Theta d z \partial_{z}\left(W_{1}^{(m)} a^{-1}\right) \partial_{z}\left(W_{1}^{\left(m^{\prime}\right)} a^{-1}\right) a^{2}, \\
I_{5}^{\left(m m^{\prime}\right)} & \equiv 2 \int d \Theta d z W_{3}^{(m)} W_{3}^{\left(m^{\prime}\right)}, \\
I_{6}^{\left(m m^{\prime}\right)} & \equiv 2 \int d \Theta d z W_{2}^{(m)} \partial_{z}\left(W_{1}^{\left(m^{\prime}\right)} a^{-1}\right) a, \\
I_{7}^{\left(m m^{\prime}\right)} & \equiv 2 \int d \Theta d z \partial_{\Theta}\left(W_{2}^{(m)} a^{-1}\right) \partial_{\Theta}\left(W_{2}^{\left(m^{\prime}\right)} a^{-1}\right) a^{2}, \\
I_{8}^{\left(m m^{\prime}\right)} & \equiv 2 \int d \Theta d z W_{3}^{(m)} \partial_{\Theta}\left(W_{1}^{\left(m^{\prime}\right)} a^{-1}\right) a, \\
I_{9}^{\left(m m^{\prime}\right)} & \equiv 2 \int d \Theta d z \partial_{z}\left(W_{3}^{(m)} a^{-1}\right) \partial_{z}\left(W_{3}^{\left(m^{\prime}\right)} a^{-1}\right) a^{2}, \\
I_{10}^{\left(m m^{\prime}\right)} & \equiv 2 \int d \Theta d z \partial_{z}\left(W_{3}^{(m)} a^{-1}\right) \partial_{\Theta}\left(W_{2}^{\left(m^{\prime}\right)} a^{-1}\right) a^{2} .
\end{aligned}
$$


By varying the four-dimensional effective action (5.19) with respect to $\hat{X}_{\mu}^{(m)}, \hat{\zeta}^{(m)}$, and $\hat{\xi}^{(m)}$, we have

$$
\begin{aligned}
& \frac{I_{1}^{\left(m m^{\prime}\right)}}{\sqrt{-\hat{g}}} \partial_{\mu_{1}}\left(\sqrt{-\hat{g}} \hat{F}^{(m) \mu_{1} \mu_{2}}\right) \\
& -\left(I_{2}^{\left(m m^{\prime}\right)}+I_{4}^{\left(m m^{\prime}\right)}\right) \hat{X}^{\mu_{2}(m)}+I_{6}^{\left(m m^{\prime}\right)} \partial^{\mu_{2}} \hat{\zeta}+I_{8}^{\left(m m^{\prime}\right)} \partial^{\mu_{2}} \hat{\xi}=0, \\
& \frac{I_{3}^{\left(m m^{\prime}\right)}}{\sqrt{-\hat{g}}} \partial_{\mu_{1}}\left(\sqrt{-\hat{g}} \partial^{\mu_{1}} \hat{\zeta}^{\left(m^{\prime}\right)}\right) \\
& -\frac{I_{6}^{\left(m m^{\prime}\right)}}{\sqrt{-\hat{g}}} \partial_{\mu_{1}}\left(\sqrt{-\hat{g}} \hat{X}^{\mu_{1}\left(m^{\prime}\right)}\right)-I_{7}^{\left(m m^{\prime}\right)} \hat{\zeta}^{\left(m^{\prime}\right)}+I_{10}^{\left(m m^{\prime}\right)} \hat{\xi}^{\left(m^{\prime}\right)}=0, \\
& \frac{I_{5}^{\left(m m^{\prime}\right)}}{\sqrt{-\hat{g}}} \partial_{\mu_{1}}\left(\sqrt{-\hat{g}} \partial^{\mu_{1}} \hat{\xi}^{\left(m^{\prime}\right)}\right) \\
& -\frac{I_{8}^{\left(m m^{\prime}\right)}}{\sqrt{-\hat{g}}} \partial_{\mu_{1}}\left(\sqrt{-\hat{g}} \hat{X}^{\mu_{1}\left(m^{\prime}\right)}\right)-I_{9}^{\left(m m^{\prime}\right)} \hat{\xi}^{\left(m^{\prime}\right)}+I_{10}^{\left(m m^{\prime}\right)} \hat{\zeta}^{\left(m^{\prime}\right)}=0 .
\end{aligned}
$$

On the other hand, by varying the six-dimensional action (5.15) with respect to $A_{M}$, we obtain the equation of motion

$$
\frac{1}{\sqrt{-g}} \partial_{M}\left(\sqrt{-g} g^{M P} g^{N Q} F_{P Q}\right)=0
$$

Eq. (5.22) can be written as

$$
\begin{aligned}
& \frac{1}{\sqrt{-\hat{g}}} \partial_{\mu_{1}}\left(\sqrt{-\hat{g}} \hat{F}^{\mu_{1} \mu_{2}(m)}\right) \\
& \quad+\left(\lambda_{1}+\lambda_{2}\right) \hat{X}^{\mu_{2}(m)}-\lambda_{4} \partial^{\mu_{2}} \hat{\zeta}^{(m)}-\lambda_{3} \partial^{\mu_{2}} \hat{\xi}^{(m)}=0 \\
& \frac{1}{\sqrt{-\hat{g}}} \partial_{\mu_{1}}\left(\sqrt{-\hat{g}} \hat{g}^{\mu_{1} \mu_{2}} \partial_{\mu_{2}} \hat{\zeta}^{(m)}\right) \\
& \quad-\lambda_{5} \frac{1}{\sqrt{-\hat{g}}} \partial_{\mu_{1}}\left(\sqrt{-\hat{g}} \hat{g}^{\mu_{1} \mu_{2}} \hat{X}_{\mu_{2}}^{(m)}\right)+\lambda_{6} \hat{\zeta}^{(m)}-\lambda_{7} \hat{\xi}^{(m)}=0 \\
& \frac{1}{\sqrt{-\hat{g}}} \partial_{\mu_{1}}\left(\sqrt{-\hat{g}} \hat{g}^{\mu_{1} \mu_{2}} \partial_{\mu_{2}} \hat{\xi}(m)\right) \\
& \quad-\lambda_{8} \frac{1}{\sqrt{-\hat{g}}} \partial_{\mu_{1}}\left(\sqrt{-\hat{g}} \hat{g}^{\mu_{1} \mu_{2}} \hat{X}_{\mu_{2}}^{(m)}\right)-\lambda_{9} \hat{\zeta}^{(m)}+\lambda_{10} \hat{\xi}^{(m)}=0
\end{aligned}
$$

where

$$
\begin{array}{ll}
\lambda_{1} \equiv \frac{\partial_{\Theta}\left(a^{2}\left(\partial_{\Theta}\left(W_{1}^{(m)} a^{-1}\right)\right)\right) a^{-1}}{W_{1}^{(m)}}, & \lambda_{2} \equiv \frac{\partial_{z}\left(a^{2} \partial_{z}\left(W_{1}^{(m)} a^{-1}\right)\right) a^{-1}}{W_{1}^{(m)}} \\
\lambda_{3} \equiv \frac{\partial_{\Theta}\left(W_{3}^{(m)} a\right) a^{-1}}{W_{1}^{(m)}}, & \lambda_{4} \equiv \frac{\partial_{z}\left(W_{2}^{(m)} a\right) a^{-1}}{W_{1}^{(m)}} \\
\lambda_{5} \equiv \frac{\partial_{z}\left(W_{1}^{(m)} a^{-1}\right) a}{W_{2}^{(m)}}, & \lambda_{6} \equiv \frac{\partial_{\Theta}\left(\partial_{\Theta}\left(W_{2}^{(m)} a^{-1}\right) a^{2}\right) a^{-1}}{W_{2}^{(m)}}
\end{array}
$$




$$
\begin{aligned}
\lambda_{7} \equiv \frac{\partial_{\Theta}\left(\partial_{z}\left(W_{3}^{(m)} a^{-1}\right) a^{2}\right) a^{-1}}{W_{2}^{(m)}}, & \lambda_{8} \equiv \frac{\partial_{\Theta}\left(W_{1}^{(m)} a^{-1}\right) a}{W_{3}^{(m)}}, \\
\lambda_{9} \equiv \frac{\partial_{z}\left(\partial_{\Theta}\left(W_{2}^{(m)} a^{-1}\right) a^{2}\right) a^{-1}}{W_{3}^{(m)}}, & \lambda_{10} \equiv \frac{\partial_{z}\left(\partial_{z}\left(W_{3}^{(m)} a^{-1}\right) a^{2}\right) a^{-1}}{W_{3}^{(m)}} .
\end{aligned}
$$

Note that we have not required $\lambda_{i}(i=1,2, \cdots, 10)$ to be constants yet.

Eq. (5.21) derived from the four-dimensional effective action (5.19) needs to be compatible with eq. (5.23) from the six-dimensional one (5.15), which leads to the following consistency conditions:

$$
\begin{array}{llll}
I_{1}^{\left(m m^{\prime}\right)}=\delta^{m m^{\prime}}, & I_{2}^{\left(m m^{\prime}\right)}=-\lambda_{1} \delta^{m m^{\prime}}, & I_{4}^{\left(m m^{\prime}\right)}=-\lambda_{2} \delta^{m m^{\prime}}, & I_{6}^{\left(m m^{\prime}\right)}=-\lambda_{4} \delta^{m m^{\prime}}, \\
I_{8}^{\left(m m^{\prime}\right)}=-\lambda_{3} \delta^{m m^{\prime}}, & I_{3}^{\left(m m^{\prime}\right)}=\delta^{m m^{\prime}}, & I_{6}^{\left(m m^{\prime}\right)}=\lambda_{5} \delta^{m m^{\prime}}, & I_{7}^{\left(m m^{\prime}\right)}=-\lambda_{6} \delta^{m m^{\prime}}, \\
I_{10}^{\left(m m^{\prime}\right)}=-\lambda_{7} \delta^{m m^{\prime}}, & I_{5}^{\left(m m^{\prime}\right)}=\delta^{m m^{\prime}}, & I_{8}^{\left(m m^{\prime}\right)}=\lambda_{8} \delta^{m m^{\prime}}, & I_{9}^{\left(m m^{\prime}\right)}=\lambda_{10} \delta^{m m^{\prime}}, \\
I_{10}^{\left(m m^{\prime}\right)}=\lambda_{9} \delta^{m m^{\prime}} . & &
\end{array}
$$

Requiring that $\lambda_{i}(i=1,2, \cdots, 10)$ are finite constants is a necessary condition but not sufficient for the consistency conditions (5.25). Furthermore, eq. (5.24) can be regarded as the equations by separating variables for eq. (5.23).

These conditions guarantee the consistency between the fundamental six-dimensional theory and the four-dimensional effective one. Starting from the effective action, one should get compatible results with the fundamental six-dimensional theory. On the other hand, these conditions impose constraints on the higher-dimensional model to ensure that the higher-dimensional theory cannot be incompatible to observations.

We further separate variables as

$$
\begin{aligned}
& W_{1}^{(m)}(z, \Theta)=\sum_{n} w_{1}^{(m, n)}(z) e^{i l_{n} \Theta}, \\
& W_{2}^{(m)}(z, \Theta)=\sum_{n} w_{2}^{(m, n)}(z) e^{i l_{n} \Theta}, \\
& W_{3}^{(m)}(z, \Theta)=\sum_{n} w_{3}^{(m, n)}(z) e^{i l_{n} \Theta} .
\end{aligned}
$$

Then, eq. (5.24) can be rewritten as

$$
\begin{aligned}
\lambda_{1} & =-l_{n}{ }^{2}, & \lambda_{2} w_{1}^{(m, n)} & =\partial_{z}\left(a^{2} \partial_{z}\left(w_{1}^{(m, n)} a^{-1}\right)\right) a^{-1}, \\
\frac{\lambda_{3}}{i l_{n}} w_{1}^{(m, n)} & =w_{3}^{(m, n)}, & \lambda_{4} w_{1}^{(m, n)} & =\partial_{z}\left(w_{2}^{(m, n)} a\right) a^{-1}, \\
\lambda_{5} w_{2}^{(m, n)} & =\partial_{z}\left(w_{1}^{(m, n)} a^{-1}\right) a, & \lambda_{6} & =-l_{n}^{2}, \\
\lambda_{7} w_{2}^{(m, n)} & =i l_{n} \partial_{z}\left(w_{3}^{(m, n)} a^{-1}\right) a, & \frac{\lambda_{8}}{i l_{n}} w_{3}^{(m, n)} & =w_{1}^{(m, n)}, \\
\lambda_{9} w_{3}^{(m, n)} & =i l_{n} \partial_{z}\left(w_{2}^{(m, n)} a\right) a^{-1}, & \lambda_{10} w_{3}^{(m, n)} & =\partial_{z}\left(\partial_{z}\left(w_{3}^{(m, n)} a^{-1}\right) a^{2}\right) a^{-1} .
\end{aligned}
$$




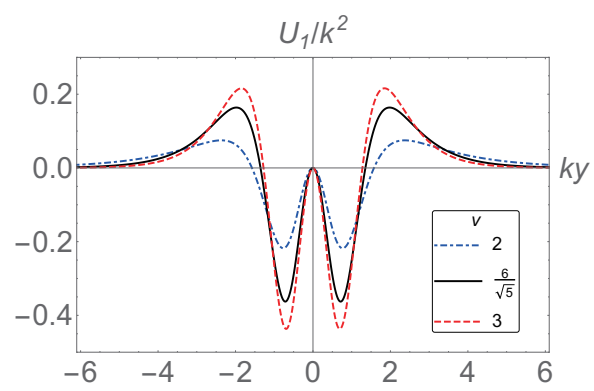

(a) The effective potential of the Schrödinger-like equation.

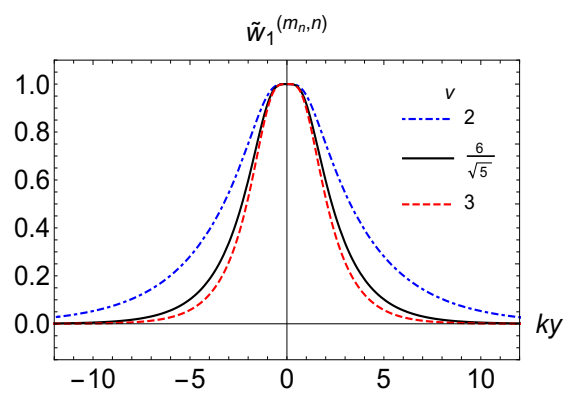

(b) The wave function of the ground state with the eigenvalue $m_{n}^{2}-l_{n}^{2}=0$.

Figure 8. The effective potential and the wave function of the ground state of eq. (5.31).

By canonically normalizing the action (5.19), the four-dimensional effective action can be written as

$$
S=-\frac{1}{4} \sum_{m} \sum_{m^{\prime}} \int d^{4} x \sqrt{-\hat{g}}\left(\hat{F}_{\mu_{1} \mu_{2}}^{(m)} \hat{F}^{\mu_{1} \mu_{2}\left(m^{\prime}\right)}+\frac{I_{2}^{\left(m m^{\prime}\right)}+I_{4}^{\left(m m^{\prime}\right)}}{I_{1}^{\left(m m^{\prime}\right)}} \hat{X}_{\mu_{1}}^{(m)} \hat{X}^{\mu_{1}\left(m^{\prime}\right)}+\cdots\right) .
$$

Taking into account the consistency conditions (5.25), we have

$$
\lambda_{1}+\lambda_{2}=-\frac{I_{2}^{\left(m m^{\prime}\right)}+I_{4}^{\left(m m^{\prime}\right)}}{I_{1}^{\left(m m^{\prime}\right)}},
$$

the effective mass $m$ is given by

$$
m^{2}=\lambda_{1}+\lambda_{2}
$$

Substituting $\lambda_{1}$ and $\lambda_{2}$ defined in (5.24) into eq. (5.29), we obtain

$$
\left[-\partial_{z}^{2}+U_{1}(z)\right] w_{1}^{(m, n)}=\left(m^{2}-l_{n}^{2}\right) w_{1}^{(m, n)},
$$

where the effective potential $U_{1}(z)$ is

$$
U_{1}(z)=\frac{\partial_{z}^{2} a}{a}
$$

which is shown in figure 8(a). The Schrödinger-like equation (5.31) can be factorized as

$$
\left(\partial_{z}+\frac{\partial_{z} a}{a}\right)\left(-\partial_{z}+\frac{\partial_{z} a}{a}\right) w_{1}^{(m, n)}=\left(m^{2}-l_{n}^{2}\right) w_{1}^{(m, n)} .
$$

The above equation possesses the form as $B^{\dagger} B W_{1}^{(m, n)}=\left(m^{2}-l_{n}^{2}\right) W_{1}^{(m, n)}$ with $B=-\partial_{z}+$ $\left(\partial_{z} a\right) / a$, which shows that $m^{2}-l_{n}^{2} \geqslant 0$ for the warp factor considered in this paper and hence there is no tachyon state.

The equation (5.33) has the massless s-wave solution $w_{1}^{(0,0)}(z)=C_{3} a(z)$, where $C_{3}$ is a constant. To obtain the four-dimensional Maxwell electromagnetic theory, the $w_{1}^{(0,0)}(z)$ 
should be normalizable. According to the consistency conditions (5.25), the normalization condition for the massless s-wave mode is

$$
I_{1}^{(00)}=\int_{-\infty}^{+\infty}\left|w_{1}^{(0,0)}(z)\right|^{2} d z=\int_{-\infty}^{+\infty}\left|w_{1}^{(0,0)}(y)\right|^{2} a^{-1}(y) d y=C_{3}^{2} \int_{-\infty}^{+\infty} a(y) d y=1
$$

From eq. (4.18), we find that the normalization condition can be guaranteed. Hence the massless s-wave mode can be localized on the brane and the four-dimensional Maxwell electromagnetic theory can be recovered.

In addition, substituting the definitions (5.20) and (5.24) into the consistency conditions (5.25), we find that the consistency conditions (5.25) have no contradiction with each other in this model.

\section{Conclusions and discussions}

Using a real scalar field as the dynamic field, we obtained two analytical solutions of a smooth thick brane with a compact extra dimension and an infinite one. The bulk is an asymptotically $\mathrm{AdS}_{6}$ spacetime and is conformally flat. Furthermore, the spacetime is stable under the tensor perturbations in the linear order, and the Newtonian potential on the brane can be recovered. In fact, our model can be regarded as a six-dimensional extension of the RS-2 model. Similar to the motivation of the RS-2 model, we achieved the localization of the graviton in the case with an infinite extra dimension and a compact one. But the free $\mathrm{U}(1)$ gauge field is also localized. This is an advantage compared with the five-dimensional thick brane extension of the RS-2 model.

With the background solution, we analyzed the tensor perturbations. Through the KK decomposition of the higher-dimensional graviton, we found that the massless s-wave mode can be localized on the brane. Phenomenologically, the Newtonian gravity is recovered on the brane. The massive KK gravitons will give a correction to the Newtonian potential. The correction includes the contribution of the continuous spectrum (like the RS-2 model) and the discrete one. Different from the five-dimensional RS-2 model, there are a series of localized massive modes of the graviton on the brane in our model. The experimental verification of the inverse-square law requires that $R_{0}$ should be less than sub-millimeter.

The localization of the massless scalar field is similar to that of gravity, and we interpreted the localization picture through two equivalent viewpoints, i.e., the curved spacetime viewpoint and the conformal flat spacetime viewpoint. The splitting of the brane may imply the splitting of the effective potentials of the KK modes of matter fields [36, 73-78]. The calculation in this paper shows that the four-dimensional zero-mass modes of the gravitational field, scalar field and gauge field are still localized near $z=0$, although the effective potentials split for solution 2. By comparing solution 1, it can be found that the zero modes are localized over a wider area along the extra dimension for solution 2 . Through the analysis of the supersymmetric partner potentials of the effective potentials, the resonant states of matter fields may occur in solution 2 scenario. These will lead to new physical effects which will help us to test this model. Further research could be an interesting subject. 
Further, we studied the localization of the free scalar field and the free U(1) gauge field. The spacetime geometry ensures that not only the scalar field but also the free $\mathrm{U}(1)$ gauge field can be localized on the brane. Following the method in ref. [58], we proved that there is no contradiction among the consistency conditions in our model. It means that a fourdimensional free $\mathrm{U}(1)$ gauge field theory can be obtained by reducing the six-dimensional action. Localization of the free U(1) gauge field implies that the four-dimensional Maxwell electromagnetic interaction can also be recovered on the brane. The KK modes of the U(1) gauge field will make contributions to the Coulomb potential [79], which deserves further study. In principle, the free $\mathrm{U}(1)$ field can be localized on the brane for a $D$-dimensional model including compact extra dimensions.

Besides the localization of boson fields, the localization of fermion fields is still worth to explore. Whenever the dimension of the momentum space increases by two, the dimension of the spinor space will double. Different from the five-dimensional case, the structure of spinors will be more abundant in six-dimensional spacetime.

\section{Acknowledgments}

We thank Wen-Di Guo, Tao-Tao Sui, Ke-Yang, Bao-Min Gu, Yi Zhong, Hao Yu, Yu-Qiang Liu, Qin Tan, especially Yu-Peng Zhang and Chun-E Fu for many useful discussions. This work is supported in part by the National Key Research and Development Program of China ("Grant No. 2020YFC2201503), the National Natural Science Foundation of China (Grants No. 11875151 and No. 12047501), and the 111 Project (Grant No. B20063).

Open Access. This article is distributed under the terms of the Creative Commons Attribution License (CC-BY 4.0), which permits any use, distribution and reproduction in any medium, provided the original author(s) and source are credited.

\section{References}

[1] N. Arkani-Hamed, S. Dimopoulos and G.R. Dvali, The hierarchy problem and new dimensions at a millimeter, Phys. Lett. B 429 (1998) 263 [hep-ph/9803315] [InSPIRE].

[2] I. Antoniadis, N. Arkani-Hamed, S. Dimopoulos and G.R. Dvali, New dimensions at a millimeter to a Fermi and superstrings at a TeV, Phys. Lett. B 436 (1998) 257 [hep-ph/9804398] [INSPIRE].

[3] L. Randall and R. Sundrum, A large mass hierarchy from a small extra dimension, Phys. Rev. Lett. 83 (1999) 3370 [hep-ph/9905221] [INSPIRE].

[4] L. Randall and R. Sundrum, An alternative to compactification, Phys. Rev. Lett. 83 (1999) 4690 [hep-th/9906064] [INSPIRE].

[5] S. Weinberg, The cosmological constant problem, Rev. Mod. Phys. 61 (1989) 1 [INSPIRE].

[6] J.E. Kim, B. Kyae and H.M. Lee, Randall-Sundrum model for selftuning the cosmological constant, Phys. Rev. Lett. 86 (2001) 4223 [hep-th/0011118] [InSPIRE].

[7] T. Kaluza, Zum Unitätsproblem der Physik, Sitzungsber. Preuss. Akad. Wiss. Berlin (Math. Phys. ) 1921 (1921) 966 [arXiv:1803.08616] [INSPIRE]. 
[8] O. Klein, Quantum theory and five-dimensional theory of relativity (in German and English), Z. Phys. 37 (1926) 895 [inSPIRE].

[9] V.A. Rubakov and M.E. Shaposhnikov, Do we live inside a domain Wall?, Phys. Lett. B 125 (1983) 136 [INSPIRE].

[10] K. Akama, An early proposal of 'brane world', Lect. Notes Phys. 176 (1982) 267 [hep-th/0001113] [INSPIRE].

[11] C. Csáki, J. Erlich, T.J. Hollowood and Y. Shirman, Universal aspects of gravity localized on thick branes, Nucl. Phys. B 581 (2000) 309 [hep-th/0001033] [INSPIRE].

[12] M. Gremm, Four-dimensional gravity on a thick domain wall, Phys. Lett. B 478 (2000) 434 [hep-th/9912060] [INSPIRE].

[13] T. Gherghetta and A. Pomarol, Bulk fields and supersymmetry in a slice of AdS, Nucl. Phys. $B 586$ (2000) 141 [hep-ph/0003129] [INSPIRE].

[14] O. Arias, R. Cardenas and I. Quiros, Thick brane worlds arising from pure geometry, Nucl. Phys. B 643 (2002) 187 [hep-th/0202130] [INSPIRE].

[15] V.I. Afonso, D. Bazeia and L. Losano, First-order formalism for bent brane, Phys. Lett. B 634 (2006) 526 [hep-th/0601069] [inSPIRE].

[16] H. Liu, H. Lü and Z.-L. Wang, $f(R)$ gravities, Killing spinor equations, 'BPS' domain walls and cosmology, JHEP 02 (2012) 083 [arXiv:1111.6602] [INSPIRE].

[17] K. Agashe, A. Azatov, Y. Cui, L. Randall and M. Son, Warped dipole completed, with a tower of Higgs bosons, JHEP 06 (2015) 196 [arXiv: 1412.6468] [INSPIRE].

[18] O. DeWolfe, D.Z. Freedman, S.S. Gubser and A. Karch, Modeling the fifth-dimension with scalars and gravity, Phys. Rev. D 62 (2000) 046008 [hep-th/9909134] [INSPIRE].

[19] T. Shiromizu, K.-i. Maeda and M. Sasaki, The Einstein equation on the 3-brane world, Phys. Rev. D 62 (2000) 024012 [gr-qc/9910076] [INSPIRE].

[20] M.N. Smolyakov and I.P. Volobuev, Some problems with reproducing the Standard Model fields and interactions in five-dimensional warped brane world models, Int. J. Mod. Phys. A 31 (2016) 1650001 [arXiv:1503.09074] [INSPIRE].

[21] B. Bajc and G. Gabadadze, Localization of matter and cosmological constant on a brane in anti-de Sitter space, Phys. Lett. B 474 (2000) 282 [hep-th/9912232] [INSPIRE].

[22] A. Kehagias and K. Tamvakis, Localized gravitons, gauge bosons and chiral fermions in smooth spaces generated by a bounce, Phys. Lett. B 504 (2001) 38 [hep-th/0010112] [INSPIRE].

[23] I. Oda, A new mechanism for trapping of photon, hep-th/0103052 [INSPIRE].

[24] G.R. Dvali, G. Gabadadze and M.A. Shifman, (Quasi)localized gauge field on a brane: Dissipating cosmic radiation to extra dimensions?, Phys. Lett. B 497 (2001) 271 [hep-th/0010071] [INSPIRE].

[25] B. Batell and T. Gherghetta, Yang-Mills localization in warped space, Phys. Rev. D 75 (2007) 025022 [hep-th/0611305] [INSPIRE].

[26] A. Flachi and M. Minamitsuji, Field localization on a brane intersection in anti-de Sitter spacetime, Phys. Rev. D 79 (2009) 104021 [arXiv:0903.0133] [InSPIRE]. 
[27] A.E.R. Chumbes, J.M. Hoff da Silva and M.B. Hott, A model to localize gauge and tensor fields on thick branes, Phys. Rev. D 85 (2012) 085003 [arXiv:1108.3821] [INSPIRE].

[28] W.T. Cruz, A.R.P. Lima and C.A.S. Almeida, Gauge field localization on the Bloch brane, Phys. Rev. D 87 (2013) 045018 [arXiv:1211.7355] [INSPIRE].

[29] C.A. Vaquera-Araujo and O. Corradini, Localization of Abelian gauge fields on thick branes, Eur. Phys. J. C 75 (2015) 48 [arXiv:1406.2892] [INSPIRE].

[30] G. Alencar, R.R. Landim, M.O. Tahim and R.N. Costa Filho, Gauge field localization on the brane through geometrical coupling, Phys. Lett. B 739 (2014) 125 [arXiv:1409.4396] [INSPIRE].

[31] Z.-H. Zhao, Y.-X. Liu and Y. Zhong, U(1) gauge field localization on a Bloch brane with Chumbes-Holf da Silva-Hott mechanism, Phys. Rev. D 90 (2014) 045031 [arXiv:1402.6480] [INSPIRE].

[32] Z.-H. Zhao, Q.-Y. Xie and Y. Zhong, New localization method of U(1) gauge vector field on flat branes in (asymptotic) AdS spacetime, Class. Quant. Grav. 32 (2015) 035020 [arXiv: 1406.3098] [INSPIRE].

[33] Z.-H. Zhao and Q.-Y. Xie, Localization of U(1) gauge vector field on flat branes with five-dimension (asymptotic) AdS spacetime, JHEP 05 (2018) 072 [arXiv:1712.09843] [INSPIRE].

[34] X.-N. Zhou, Y.-Z. Du, Z.-H. Zhao and Y.-X. Liu, Localization of five-dimensional Elko spinors with non-minimal coupling on thick branes, Eur. Phys. J. C 78 (2018) 493 [arXiv: 1710.02842] [INSPIRE].

[35] L.F. Freitas, G. Alencar and R.R. Landim, Universal aspects of U(1) gauge field localization on branes in D-dimensions, JHEP 02 (2019) 035 [arXiv: 1809.07197] [INSPIRE].

[36] T.-T. Sui, W.-D. Guo, Q.-Y. Xie and Y.-X. Liu, Generalized geometrical coupling for vector field localization on thick brane in asymptotic Anti-de Sitter spacetime, Phys. Rev. D 101 (2020) 055031 [arXiv:2001.02154] [INSPIRE].

[37] J.E.G. Silva, W.H.P. Brandao, R.V. Maluf and C.A.S. Almeida, Regular string-like braneworlds, Eur. Phys. J. C 79 (2019) 77 [arXiv:1711.08564] [InSPIRE].

[38] M. Giovannini, H. Meyer and M.E. Shaposhnikov, Warped compactification on Abelian vortex in six-dimensions, Nucl. Phys. B 619 (2001) 615 [hep-th/0104118] [INSPIRE].

[39] S. Kanno and J. Soda, Quasi-thick codimension 2 braneworld, JCAP 07 (2004) 002 [hep-th/0404207] [INSPIRE].

[40] J. Vinet and J.M. Cline, Can codimension-two branes solve the cosmological constant problem?, Phys. Rev. D 70 (2004) 083514 [hep-th/0406141] [InSPIRE].

[41] I. Olasagasti and A. Vilenkin, Gravity of higher dimensional global defects, Phys. Rev. D 62 (2000) 044014 [hep-th/0003300] [INSPIRE].

[42] J. Garriga and M. Porrati, Football shaped extra dimensions and the absence of self-tuning, JHEP 08 (2004) 028 [hep-th/0406158] [INSPIRE].

[43] M. Gogberashvili, P. Midodashvili and D. Singleton, Fermion generations from 'apple-shaped' extra dimensions, JHEP 08 (2007) 033 [arXiv:0706.0676] [INSPIRE].

[44] Y.-S. Duan, Y.-X. Liu and Y.-Q. Wang, Fermionic zero modes in gauge and gravity backgrounds on $T^{2}$, Mod. Phys. Lett. A 21 (2006) 2019 [hep-th/0602157] [INSPIRE]. 
[45] Y.-X. Liu, L. Zhao and Y.-S. Duan, Localization of Fermions on a string-like defect, JHEP 04 (2007) 097 [hep-th/0701010] [INSPIRE].

[46] A. Chodos and E. Poppitz, Warp factors and extended sources in two transverse dimensions, Phys. Lett. B 471 (1999) 119 [hep-th/9909199] [INSPIRE].

[47] J.F. Vazquez-Poritz, Gravity trapping domain walls from resolved branes, JHEP 09 (2002) 001 [hep-th/0111229] [INSPIRE].

[48] F. Brummer, A. Hebecker and E. Trincherini, The throat as a Randall-Sundrum model with Goldberger-Wise stabilization, Nucl. Phys. B 738 (2006) 283 [hep-th/0510113] [INSPIRE].

[49] H. Firouzjahi and S.H.H. Tye, The shape of gravity in a warped deformed conifold, JHEP 01 (2006) 136 [hep-th/0512076] [inSPIRE].

[50] T. Noguchi, M. Yamaguchi and M. Yamashita, Gravitational Kaluza-Klein modes in warped superstring compactification, Phys. Lett. B 636 (2006) 221 [hep-th/0512249] [INSPIRE].

[51] I. Navarro, Codimension two compactifications and the cosmological constant problem, JCAP 09 (2003) 004 [hep-th/0302129] [INSPIRE].

[52] E. Papantonopoulos, A. Papazoglou and V. Zamarias, Induced cosmology on a regularized brane in six-dimensional flux compactification, Nucl. Phys. B 797 (2008) 520 [arXiv: 0707.1396] [INSPIRE].

[53] I.I. Kogan, S. Mouslopoulos, A. Papazoglou and G.G. Ross, Multigravity in six-dimensions: generating bounces with flat positive tension branes, Phys. Rev. D 64 (2001) 124014 [hep-th/0107086] [INSPIRE].

[54] I. Oda, Localization of matters on a string-like defect, Phys. Lett. B 496 (2000) 113 [hep-th/0006203] [INSPIRE].

[55] I. Oda, Bosonic fields in the string-like defect model, Phys. Rev. D 62 (2000) 126009 [hep-th/0008012] [INSPIRE].

[56] T. Gherghetta, E. Roessl and M.E. Shaposhnikov, Living inside a hedgehog: higher dimensional solutions that localize gravity, Phys. Lett. B 491 (2000) 353 [hep-th/0006251] [INSPIRE].

[57] L.J.S. Sousa, C.A.S. Silva, D.M. Dantas and C.A.S. Almeida, Vector and fermion fields on a bouncing brane with a decreasing warp factor in a string-like defect, Phys. Lett. B 731 (2014) 64 [arXiv: 1402.1855] [inSPIRE].

[58] C.-E. Fu, Y. Zhong and Y.-X. Liu, U(1) gauge vector field on a codimension-2 brane, JHEP 01 (2019) 021 [arXiv:1810.02081] [INSPIRE].

[59] L.F.F. Freitas, G. Alencar and R.R. Landim, Consistency conditions for fields localization on braneworlds, Eur. Phys. J. C 80 (2020) 432 [arXiv:2001.01267] [INSPIRE].

[60] L.F.F. Freitas, G. Alencar and R.R. Landim, Consistency conditions for p-form field localization on codimension two braneworlds, Eur. Phys. J. C 80 (2020) 1141 [arXiv:2006.10640] [INSPIRE].

[61] M. Giovannini, Theory of gravitational fluctuations in brane world models, Int. J. Mod. Phys. D 11 (2002) 1209 [INSPIRE].

[62] M. Giovannini, Scalar normal modes of higher dimensional gravitating kinks, Class. Quant. Grav. 20 (2003) 1063 [gr-qc/0207116] [INSPIRE]. 
[63] M. Giovannini, Localization of metric fluctuations on scalar branes, Phys. Rev. D 65 (2002) 064008 [hep-th/0106131] [INSPIRE].

[64] M. Giovannini, Gauge invariant fluctuations of scalar branes, Phys. Rev. D 64 (2001) 064023 [hep-th/0106041] [INSPIRE].

[65] M. Giovannini, Gauge field localization on Abelian vortices in six-dimensions, Phys. Rev. D 66 (2002) 044016 [hep-th/0205139] [INSPIRE].

[66] D.J. Kapner et al., Tests of the gravitational inverse-square law below the dark-energy length scale, Phys. Rev. Lett. 98 (2007) 021101 [hep-ph/0611184] [INSPIRE].

[67] G.D. Kribs, TASI 2004 lectures on the phenomenology of extra dimensions, in Physics in $D \geq 4$, J. Terning et al. eds., World Scientific, Singapore (2006), hep-ph/0605325 [INSPIRE].

[68] H.-C. Cheng, Introduction to extra dimensions, in Physics of the large and the small, C. Csáki ed., World Scientific, Singapore (2010), arXiv:1003.1162 [INSPIRE].

[69] E.G. Adelberger, B.R. Heckel and A.E. Nelson, Tests of the gravitational inverse square law, Ann. Rev. Nucl. Part. Sci. 53 (2003) 77 [hep-ph/0307284] [InSPIRE].

[70] C.D. Hoyle et al., Sub-millimeter tests of the gravitational inverse-square law, Phys. Rev. D 70 (2004) 042004 [hep-ph/0405262] [INSPIRE].

[71] S.-Q. Yang et al., Test of the gravitational inverse square law at millimeter ranges, Phys. Rev. Lett. 108 (2012) 081101 [INSPIRE].

[72] J. Ke, J. Luo, Y.-J. Tan and C.-G. Shao, Influence of the residual gas damping noise in the test of the gravitational inverse-square law, Class. Quant. Grav. 37 (2020) 205008 [INSPIRE].

[73] Y.-X. Liu, J. Yang, Z.-H. Zhao, C.-E. Fu and Y.-S. Duan, Fermion localization and resonances on a de Sitter thick brane, Phys. Rev. D 80 (2009) 065019 [arXiv:0904.1785] [INSPIRE].

[74] H.-T. Li, Y.-X. Liu, Z.-H. Zhao and H. Guo, Fermion resonances on a thick brane with a piecewise warp factor, Phys. Rev. D 83 (2011) 045006 [arXiv: 1006.4240] [InSPIRE].

[75] Z.-H. Zhao, Y.-X. Liu, H.-T. Li and Y.-Q. Wang, Effects of the variation of mass on fermion localization and resonances on thick branes, Phys. Rev. D 82 (2010) 084030 [arXiv: 1004.2181] [INSPIRE].

[76] Y.-P. Zhang, Y.-Z. Du, W.-D. Guo and Y.-X. Liu, Resonance spectrum of a bulk fermion on branes, Phys. Rev. D 93 (2016) 065042 [arXiv:1601.05852] [inSPIRE].

[77] H. Yu, Y. Zhong, B.-M. Gu and Y.-X. Liu, Gravitational resonances on $f(R)$-brane, Eur. Phys. J. C 76 (2016) 195 [arXiv: 1506. 06458] [INSPIRE].

[78] Y. Zhong, Y.-P. Zhang, W.-D. Guo and Y.-X. Liu, Gravitational resonances in mimetic thick branes, JHEP 04 (2019) 154 [arXiv: 1812.06453] [INSPIRE].

[79] H. Guo, A. Herrera-Aguilar, Y.-X. Liu, D. Malagon-Morejon, and R. R. Mora-Luna, Localization of bulk matter fields, the hierarchy problem and corrections to Coulomb's law on a pure de Sitter thick braneworld, Phys. Rev. D 87 (2013) 095011 [arXiv:1103.2430] [INSPIRE]. 